\title{
A combined state-space identification algorithm applied to data from a modal analysis experiment on a separation system
}

\author{
Tomas McKelvey \\ Department of Electrical Engineering \\ Linköping University \\ S-581 83 Linköping, Sweden \\ e-mail: Tomas.McKelvey@isy.liu.se \\ Fax: +4613282622
}

February 23, 1994

\section{3rd CDC}

\begin{abstract}
This paper discuss identification of state-space models from impulse response or initial value experiments. We combine Kung's realization algorithm with classical nonlinear parametric optimization in order to further refine the quality of the estimated state-space model. We apply these ideas on real data originating from a modal analysis experiment on a separation system. The results indicate that the parametric optimization step increase the model quality significantly compared with the initial model the realization algorithm provides.
\end{abstract}

Keywords: system identification, modal analysis, state-space methods, application.

\section{Introduction}

Identification of modal parameters of flexible mechanical structures can be carried out by estimating a state-space model given impulse response or initial value response measurements, see e.g. [6, 2, 4].

Consider the state-space model

$$
\begin{aligned}
x(t+1) & =A x(t), x(0)=x 0 \\
y(t) & =C x(t)
\end{aligned}
$$

where $y(t) \in \mathbb{R}^{p}$ and $x(t) \in \mathbb{R}^{n}$ with $n$ equal the order of the system. Given data $y_{t}, t=$ $0, \ldots, N-1$ from an initial value experiment the identification should yield estimates on $A, C$ and $x 0$. such that

$$
V(A, C, x 0)=\frac{1}{N} \sum_{t=0}^{N-1}\left|y_{t}-y(t)\right|^{2}
$$

is minimized. From the state-space model (1) it is easy to derive frequencies and damping ratio for the different structural modes. 
In this paper we present a combined algorithm which as a first step use Kung's realization algorithm [3] to obtain an initial estimate of a state space system matching the data. In a second step an initial state $x 0$ is estimated by a linear least-squares fit to the data. In the last step a nonlinear optimization is performed to further improve the criterion (2). The first step is closely related to [2] since both algorithms rely on the realization algorithm by Ho \& Kalman [1]. The second and last steps are introduced since the estimates from the first step in general do not minimize (2) if the true system is of high order and/or if noise is present.

We apply the combined algorithm to real data from a modal analysis experiment of a separation system. A separation system is the connecting device between the launcher and the payload and serves the purpose of keeping the payload in place, on top of the launcher, during the launch phase and when reached the correct orbit, release the payload from the launcher. During the separation phase an explosive charge in the separation system release the payload. In order to reduce oscillations on the payload the separation system is designed to dampen out oscillations. The real data origin from a separation experiment where oscillations were measured with three accelerometers. The interesting parameters to extract are frequencies and damping ratio for the dominating modes. The data has been made available to the author by courtesy of Saab Ericsson Space AB, Linköping, Sweden.

\section{A combined identification algorithm}

The first estimate is obtained by using Kung's algorithm [3]: Assume we are given $N=q+r-1$ noise free measurements $y_{t}$. Construct the block-hankel matrix

$$
Y_{q r}=\left[\begin{array}{cccc}
y_{0} & y_{1} & \cdots & y_{r} \\
y_{1} & y_{2} & \cdots & \vdots \\
\vdots & \vdots & \vdots & \vdots \\
y_{q-2} & y_{q-1} & \cdots & y_{q+r-2}
\end{array}\right]
$$

where $q>n$ and $r \geq n$ is the number of block rows and columns respectively. From (1) it follows that $y_{t}=C A^{t-1} x 0$ and hence $Y_{q r}$ can be factored as

$$
Y_{q r}=\mathcal{O}_{q} \mathcal{C}_{r}
$$

where $\mathcal{O}_{q}$ is the extended observability matrix

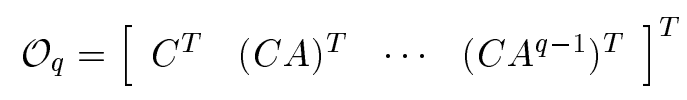

with rank $n$ if the system is observable and

$$
\mathcal{C}_{r}=\left[\begin{array}{llll}
x 0 & A x 0 & \cdots & A^{r-1} x 0
\end{array}\right]
$$

is a matrix of rank $n$ if the initial state $x 0$ excites all modes. Calculate the singular value decomposition

$$
Y_{q r}=\left[\begin{array}{ll}
\hat{U}_{1} & \hat{U}_{2}
\end{array}\right]\left[\begin{array}{cc}
\hat{\Sigma}_{1} & 0 \\
0 & \hat{\Sigma}_{2}
\end{array}\right]\left[\begin{array}{c}
V_{1}^{T} \\
V_{2}^{T}
\end{array}\right]
$$


where the diagonal matrix $\hat{\Sigma}_{1}$ contains the $n$ principal singular values. Since we assumed $y_{t}$ originated from (1), $Y_{q r}$ will be of rank $n$ if $\min (q, r)>n$ and hence $\hat{\Sigma}_{2}=0$. Let us denote

$$
\begin{aligned}
& J_{1}=\left[\begin{array}{ll}
I_{(q-1) p} & 0_{(q-1) p \times p}
\end{array}\right] \\
& J_{2}=\left[\begin{array}{ll}
0_{(q-1) p \times p} & I_{(q-1) p}
\end{array}\right]
\end{aligned}
$$

where $I_{i}$ denotes the $i \times i$ identity matrix and $0_{i \times j}$ denotes the $i \times j$ zero matrix. The system matrices are estimated as

$$
\begin{gathered}
\hat{A}_{0}=\left(J_{1} \hat{U}_{1} \hat{\Sigma}_{1}^{1 / 2}\right)^{\dagger}\left(J_{2} \hat{U}_{1} \hat{\Sigma}_{1}^{1 / 2}\right) \\
\hat{C}_{0}=\left[\begin{array}{ll}
I_{p} & 0_{p \times(q-1)}
\end{array}\right] \hat{U}_{1} \hat{\Sigma}_{1}^{1 / 2}, \\
\hat{x} 0_{0}=\hat{\Sigma}_{1}^{1 / 2} \hat{V}_{1}^{T}\left[\begin{array}{ll}
1 & 0_{1 \times(r-1)}
\end{array}\right]^{T}
\end{gathered}
$$

where $A^{\dagger}=\left(A^{T} A\right)^{-1} A^{T}$ denotes the Moore-Penrose pseudo-inverse of the full-rank matrix $A$. If the measurements $y_{t}$ are noise free the estimated system $\left(\hat{A}_{0}, \hat{C}_{0}, \hat{x} 0_{0}\right)$ are then related to the original system $(A, C, x 0)$ by a similarity transformation. If we use this algorithm on noisy data or data from a high order system $\Sigma_{2}$ will not be identically zero and $\left(\hat{A}_{0}, \hat{C}_{0}, \hat{x 0_{0}}\right)$ will then be an approximation of the true system. There is no proof that this approximation minimize (2). However, in general Kung's algorithm provide models which are close to the minimum and hence we will use this estimate as an initial estimate in solving (2) using a Gauss-Newton type optimization. As an intermediate step a refined estimate of $x 0, \hat{x} 0_{1}$ is calculated by minimizing (2) with respect to $x 0$. This minimization is easily solved by a linear least-square solution since $x 0$ is linear in $y(t)$ if $\hat{A}_{0}$ and $\hat{C}_{0}$ are held constant. In the last step $\left(\hat{A}_{0}, \hat{C}_{0}, \hat{x} 0_{1}\right)$ are converted to an observability canonical form by the use of a similarity transformation, see [5], and are then used as an initial estimate minimizing $(2)$ with respect to $(A, C, x 0)$ to obtain a final model $(\hat{A}, \hat{C}, \hat{x} 0)$. The conversion to a canonical form allows us to use a sparse parameterization of the $A$ matrix and a fix $C$ matrix and hence save computation time. Furthermore this parameterization is also identifiable and hence each point in the parameter space represents a unique system.

The estimated model is now an abstract model of the experimental data. Particularly, the eigenvalues of the matrix $\hat{A}$ give us information about frequency and damping ratio for all identified structural modes. Denote sampling frequency with $f$ given in Herz and $\lambda_{i}(A), i=1, \ldots, n$ the eigenvalues of $A$. Each structural mode $k$ with the principal shape

$$
m_{k}(t)=e^{-\zeta_{k} \Omega_{k}} \sin \left(\omega_{k} t\right)
$$

with $\Omega_{k}=\omega_{k} / \sqrt{1-\zeta^{2}}$, is characterized by the frequency $\omega_{k}$ (in $\mathrm{rad} / \mathrm{s}$ ) and relative damping ratio $\zeta_{k}$. These modal parameters are associated with a complex conjugate eigenvalue pair from the real matrix $A$ such that

$$
\begin{gathered}
\Omega_{k}=\operatorname{Im}\left(\log \lambda_{k}\right) f \\
\zeta_{k}=-\operatorname{Re}\left(\log \lambda_{k}\right) f / \Omega_{k} \\
\omega_{k}=\Omega_{k} \sqrt{1-\zeta^{2}}
\end{gathered}
$$

where $\lambda_{k}$ is the eigenvalue with positive imaginary part of the two complex conjugate eigenvalues. In order to rank the estimated modes we will apply the following procedure. Assume $\hat{A}$ has distinct 


\begin{tabular}{|c|c|c|}
\hline Frequency $(\mathrm{Hz})$ & Damping ratio (\%) & Modal influence \\
\hline \hline 1116 & 1.73 & 0.0914 \\
\hline 1129 & 0.38 & 0.0017 \\
\hline 1138 & 2.92 & 0.2317 \\
\hline 1186 & 2.31 & 0.1559 \\
\hline 1235 & 0.66 & 0.0619 \\
\hline 1309 & 1.94 & 0.1530 \\
\hline 1344 & 1.20 & 0.1779 \\
\hline 1385 & 2.85 & 0.1202 \\
\hline 1493 & 0.82 & 0.0063 \\
\hline
\end{tabular}

Table 1: Estimated frequencies and damping ratio and corresponding modal influence for the estimated model of order 18.

eigenvalues. Find the complex similarity transformation $T$ such that $\Lambda=T^{-1} \hat{A} T$ where $\Lambda$ is a diagonal matrix with the eigenvalues on the diagonal ordered in complex conjugate pairs and let $\tilde{x} 0=T^{-1} \hat{x} 0, \tilde{C}=C T$. The output can now be written as

$$
y(t)=\sum_{k=1}^{n} \tilde{c}_{k} \tilde{x} 0_{k} \lambda_{k}^{t-1}
$$

where $\tilde{c}_{k}$ is the $k$ th column of $\tilde{C}$ and $\tilde{x} 0_{k}$ is the $k$ th element of the vector $\tilde{x} 0$. For each complex conjugate eigenvalue pair and thus each mode we can define a modal influence measure $M_{k}$

$$
M_{k}=\frac{2\left|\tilde{c}_{2 k-1} \tilde{x} 0_{2 k-1}\right|}{\sum_{i=1}^{n}\left|\tilde{c}_{i} \tilde{x} 0_{i}\right|}
$$

which is a measure the influence of each mode on the output of the system. The measure $M_{k}$ is a relative measure with $\sum_{k=1}^{n / 2} M_{k}=1$.

\section{Structural mode identification: A separation system}

The experimental data consists of a record of 4096 samples of the three accelerometers obtained with a sampling frequency $f=32768 \mathrm{~Hz}$. The data is bandpass-filtered by a 5 th order Butterworth filter with passband between 1000 and $1460 \mathrm{~Hz}$ in order to remove uninteresting frequencies. In Figure 1 the values of the criterion (2) after each identification step are plotted for some different model orders. It is quite clear that both the second and third step significantly decrease the criterion and hence the quality of the estimated model. In Table 1 the estimated frequencies, damping ratios and modal influence measure are given for an estimated model of order $n=18$. In Figure 2 a portion of the filtered accelerometer signals are plotted together with a simulation of the estimated model of order 18. The model has captured almost all the dynamics in the signals and the two curves are almost indistinguishable. 


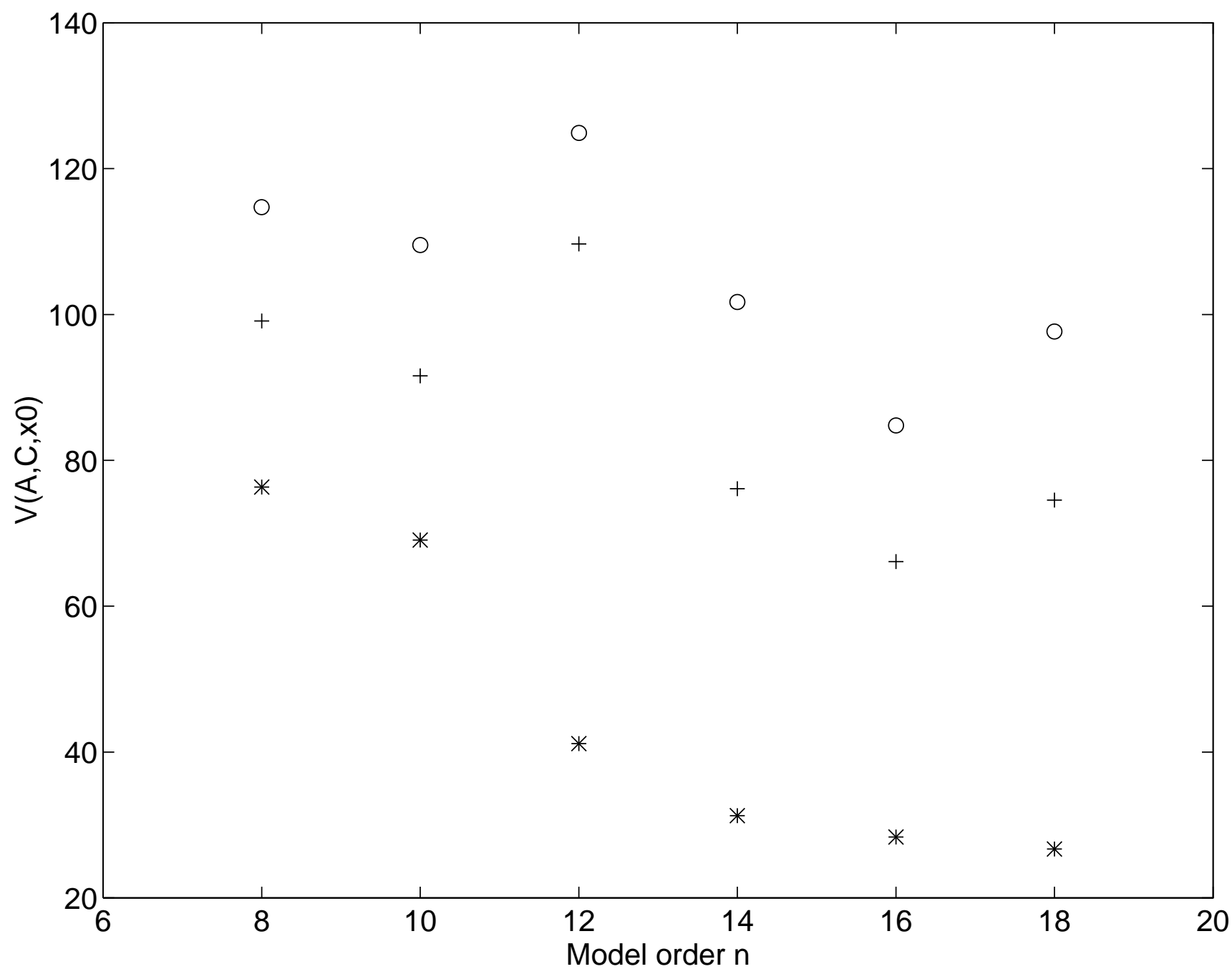

Figure 1: The criterion (2) after each step in the identification algorithm. "o" after first subspace step. "+" after x0 estimation by linear least-squares minimization. "*" after nonlinear minimization 
Accelerometer signal: 1

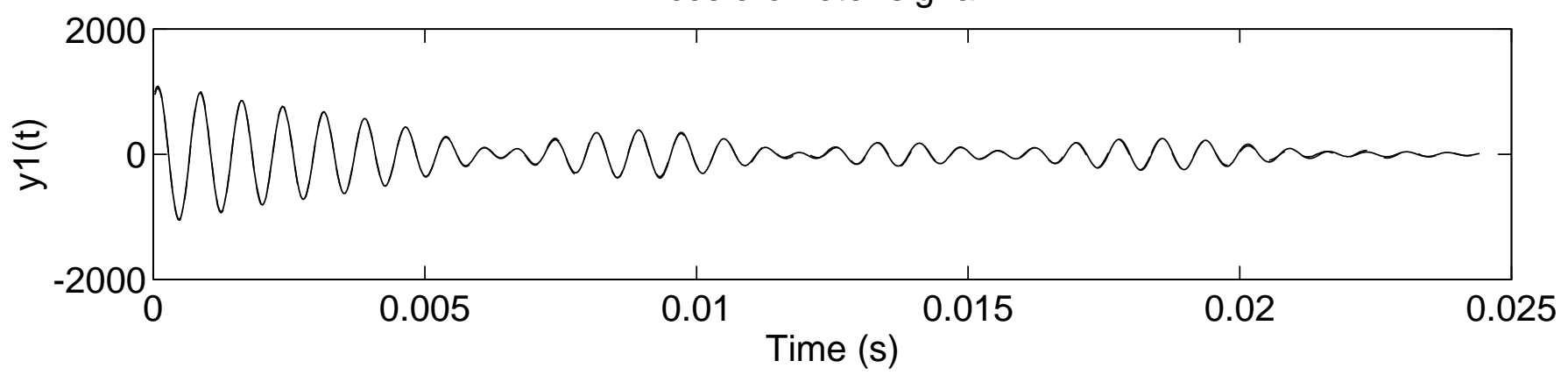

Accelerometer signal: 2

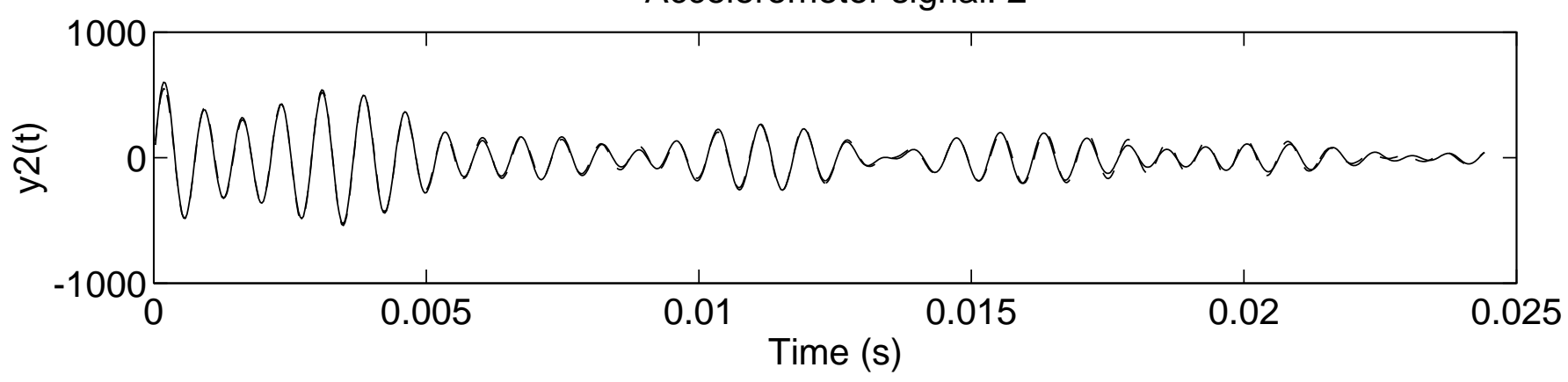

Accelerometer signal: 3

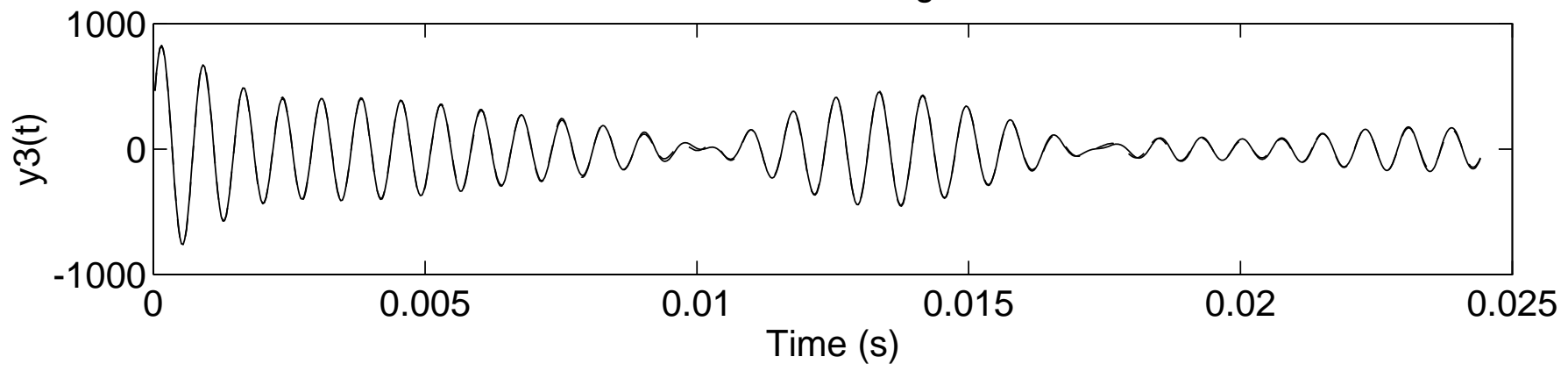

Figure 2: Comparison between measured accelerometer outputs (solid line) and simulated outputs (dashed line) using the estimated model of order 18. 


\section{References}

[1] B. L. Ho and R. E. Kalman. Effective construction of linear state-variable models from input/output functions. Regelungstechnik, 14(12):545-592, 1966.

[2] J. N. Juang and R. S. Pappa. An eigensystem realization algorithm for modal parameter identification and model reduction. J. of Guidence, Control and Dynamics, 8(5):620-627, 1985.

[3] S. Y. Kung. A new identification and model reduction algorithm via singular value decomposition. In Proc. of 12th Asilomar Conference on Circuits, Systems and Computers, Pacific Grove, $C A$, pages 705-714, 1978.

[4] J. S. Lew, J. N. Juang, and R. W. Longman. Comparison of several system identification methods for flexible structures. J. of Sound and Vibration, 167(3):461-480, 1993.

[5] L. Ljung. System Identification: Theory for the User. Prentice-Hall, Englewood Cliffs, New Jersey, 1987.

[6] H. Vold and R. Russel. Advanced analysis methods improve modal test results. Sound and Vibration, pages 36-40, 1983. 